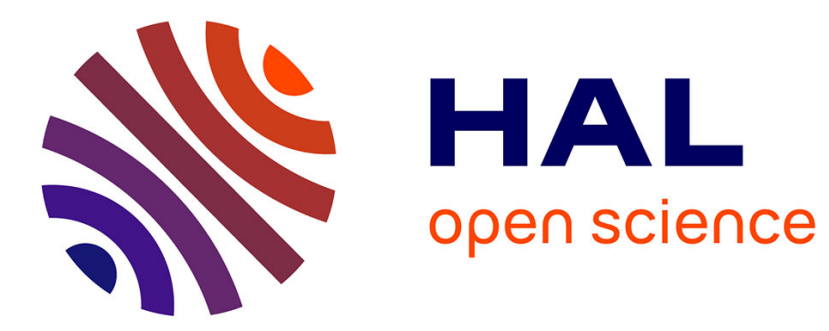

\title{
Gradual Semantics for Weighted Bipolar SETAFs
}

Bruno Yun, Srdjan Vesic

\section{To cite this version:}

Bruno Yun, Srdjan Vesic. Gradual Semantics for Weighted Bipolar SETAFs. ECSQARU The Sixteenth European Conference on Symbolic and Quantitative Approaches to Reasoning with Uncertainty (ECSQARU 2021), Sep 2021, Prague, Czech Republic. 10.1007/978-3-030-86772-0_15 . hal03426757

\section{HAL Id: hal-03426757 \\ https://hal-univ-artois.archives-ouvertes.fr/hal-03426757}

Submitted on 12 Nov 2021

HAL is a multi-disciplinary open access archive for the deposit and dissemination of scientific research documents, whether they are published or not. The documents may come from teaching and research institutions in France or abroad, or from public or private research centers.
L'archive ouverte pluridisciplinaire HAL, est destinée au dépôt et à la diffusion de documents scientifiques de niveau recherche, publiés ou non, émanant des établissements d'enseignement et de recherche français ou étrangers, des laboratoires publics ou privés. 


\title{
Gradual Semantics for Weighted Bipolar SETAFs
}

\author{
Bruno Yun ${ }^{1[0000-0001-9370-3917]}$ and Srdjan Vesic ${ }^{2, *[0000-0002-4382-0928]}$ \\ 1 University of Aberdeen, Scotland \\ bruno. yun@abdn.ac.uk \\ 2 CNRS, Univ. Artois, CRIL - Centre de Recherche en Informatique de Lens, \\ F-62300 Lens, France \\ vesic@cril.fr
}

\begin{abstract}
Gradual semantics are now well-studied in the computational argumentation literature. In this paper, we argue that gradual semantics that can handle both bipolarity (e.g. attacks and supports) and sets of attacking arguments (i.e. several arguments together attacking an argument) might be useful in some contexts. We define the formal framework and properties for such semantics. We proceed by adapting, studying and implementing three well-known semantics from the bipolar gradual literature to this new framework.
\end{abstract}

Keywords: Argumentation · Gradual semantics

\section{Introduction}

Recently, gradual argumentation semantics have drawn the attention of many scholars $[11,8,16,6,2,17,14,1]$. Some of the existing approaches for gradual semantics allow for intrinsic weights of arguments [2], some for intrinsic weights of attacks [4], some for sets of attacking arguments [22], and some for bipolarity, i.e. two types of interactions between argument are allowed: attacks and supports $[7,3,17,1]$. One might need all of those features (weights on arguments and attacks, bipolarity and sets of attacking/supporting arguments) to model some situations in an intuitive manner, as illustrated by Figure 1, which is inspired from the example by Prakken [18]. We do not claim that our example cannot be modelled by simpler frameworks, but we believe that our framework allows to represent it in a natural way.

First, the intrinsic weights of the arguments might represent the confidence level of the source or the plausibility of the argument. For instance, the argument "hot" might have a greater intrinsic weight if the outside temperature is 40 degrees Celsius than if the outside temperature is 30 degrees.

*Srdjan Vesic was supported by "Responsible AI" ANR Chair in Artificial Intelligence, https://ia-responsable.eu/ 

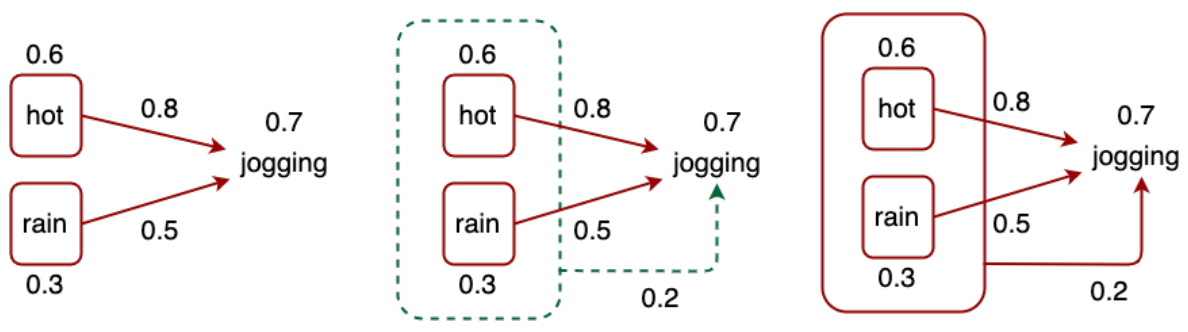

Fig. 1. Representation of the jogging example. The solid red lines represent sets of attacking arguments whereas the dashed lines represent sets of supporting arguments.

Second, the intrinsic weights on attacks represent how much arguments attack another argument. For instance, hot weather may be a good reason for cancelling a jogging whereas the rain might be less important. In Figure 1, this is represented by having a greater intrinsic weight on the attack coming from the argument "hot" (0.8) than "rain" (0.5).

Lastly, an agent might have different inclinations toward how the weather affects their jogging routine. For the sake of the example, let us model three possibilities. The simplest case is the one where both the hot weather and the rain are arguments against going for a jogging but there is no interaction between them (left part of Figure 1). The second case is the one where both the hot weather and the rain are still arguments against going for a jogging but the combination of the hot weather and the rain is a desirable reason to go for a jogging (center part of Figure 1). The third case is the one where the two factors (the hot weather and the rain) may interact together and represent a further reason against going for a jogging (right part of Figure 1).

This latter case is modelled using an argumentation framework, known in the literature, under the name of set of attacking arguments framework (SETAF). Please note that to model all three possibilities, we also need supports (in addition to attacks) to model positive and negative effects of temperature and precipitation on jogging. The reader is invited to observe the intricate link with the idea of argument accrual, which is well studied in the literature $[21,18,5,9$, $10,12,19]$. Note however, that arguments accrual was not studied for gradual semantics nor was adapted to sets of attacking argument frameworks.

The contributions of this paper are: (1) a new argumentation framework with sets of attacking/supporting arguments with intrinsic weights on relations, (2) the definition of properties for this framework and (3) the definition and characterisation of bipolar gradual semantics tailored for this framework.

The paper is structured as follows. We start by presenting the background notions and a list of desirable properties that a gradual semantics for weighted bipolar SETAFs should satisfy. We then show some links between those properties (e.g. some properties imply others). We continue by adapting three semantics from the literature (namely, Euler-based, DF-Quad and Sigmoid damped max-based semantics) to our framework and execute a formal study of their 
properties. We also provide a JAVA implementation for several semantics and extend the existing ASPARTIX format to the case of weighted bipolar SETAFs.

\section{Formal Setting}

We first extend the argumentation framework proposed by [15] with sets of supporting arguments which allow arguments to jointly support an argument.

Definition 1. A weighted bipolar SETAF (WBSETAF) is $\mathcal{A S}=\langle\mathcal{A}, \mathcal{P}, w\rangle$, where $\mathcal{A}$ is a finite set of arguments, $\mathcal{P} \subseteq\left(2^{\mathcal{A}} \backslash \emptyset\right) \times \mathcal{A} \times\{$ att, supp $\}$ is a set of collective interactions (each interaction being either an attack or a support), and $w$ is a function from $\mathcal{A} \cup \mathcal{P}$ to $[0,1]$.

The set of all WBSETAFs is denoted by $\mathcal{X}_{\text {setaf }}$. The set of non-maximal WBSETAFs is defined as $\mathcal{X}_{\text {setaf }}^{<1}=\left\{\langle\mathcal{A}, \mathcal{P}, w\rangle \in \mathcal{X}_{\text {setaf }} \mid\right.$ for every $\left.b \in \mathcal{A}, w(b)<1\right\}$ and the set of non-minimal WBSETAFs is defined as $\mathcal{X}$ setaf $=\{\langle\mathcal{A}, \mathcal{P}, w\rangle \in$ $\mathcal{X}_{\text {setaf }} \mid$ for every $\left.b \in \mathcal{A}, w(b)>0\right\} .(X, j, a t t) \in \mathcal{P}$ means that there is an attack from $X \in\left(2^{\mathcal{A}} \backslash \emptyset\right)$ to $j \in \mathcal{A}$ whereas $(X, j$, supp $) \in \mathcal{P}$ means that there is a support from $X$ to $j$. Please note that in our formal setting, we can have both an attack and a support from the same set of arguments toward an argument but all the properties in this paper still hold in the restricted case, where there is only one type of interaction (support or attack) between a set of arguments and an argument. If $a \in \mathcal{A}$, we denote by $w(a)$ the intrinsic weight of argument $a$ representing its trustworthiness or the certainty degree of the argument's premises. For $p \in \mathcal{P}$, we denote by $w(p)$ the intrinsic weight of the attack/support $p$. The set of attackers of $a \in \mathcal{A}$ is $\operatorname{Att}(a)=\left\{X \in 2^{\mathcal{A}} \mid(X, a, a t t) \in \mathcal{P}\right\}$. Likewise, the set of supports of $a \in \mathcal{A}$ is $\operatorname{Supp}(a)=\left\{X \in 2^{\mathcal{A}} \mid(X, a\right.$, supp $\left.) \in \mathcal{P}\right\}$. The union of two WBSETAFs $\mathcal{A S}=\langle\mathcal{A}, \mathcal{P}, w\rangle$ and $\mathcal{A S}^{\prime}=\left\langle\mathcal{A}^{\prime}, \mathcal{P}^{\prime}, w^{\prime}\right\rangle$ s.t. $\mathcal{A} \cap \mathcal{A}^{\prime}=\emptyset$ is $\mathcal{A S} \oplus \mathcal{A S}^{\prime}=\left\langle\mathcal{A} \cup \mathcal{A}^{\prime}, \mathcal{P} \cup \mathcal{P}^{\prime}, w^{\prime \prime}\right\rangle$, where $\forall a \in \mathcal{A} \cup \mathcal{A}^{\prime} \cup \mathcal{P} \cup \mathcal{P}^{\prime}, w^{\prime \prime}(a)=w(a)$, if $a \in \mathcal{A} \cup \mathcal{P}$ and $w^{\prime \prime}(a)=w^{\prime}(a)$, if $a \in \mathcal{A}^{\prime} \cup \mathcal{P}^{\prime}$.

A path is a sequence of sets of arguments that are linked with either sets of attacking or supporting arguments.

Definition 2 (Path). A sequence $\left\langle X_{0}, X_{1}, \ldots, X_{n}\right\rangle$ is called a path in $\mathcal{A S}=$ $\langle\mathcal{A}, \mathcal{P}, w\rangle$ iff $\forall i \in\{0, \ldots, n-1\}, \exists x \in X_{i+1}$ s.t. $\left(X_{i}, x, l\right) \in \mathcal{P}$, where $l \in$ $\{$ att, supp $\}$.

A path is significant w.r.t. a WBSETAF $\mathcal{A S}=\langle\mathcal{A}, \mathcal{P}, w\rangle$ if it is, roughly speaking, composed of attacks/supports having strictly positive weights.

Definition 3 (Significant path). A path $\left\langle X_{0}, X_{1}, \ldots, X_{n}\right\rangle$ in $\mathcal{A S}=\langle\mathcal{A}, \mathcal{P}, w\rangle$ is significant if and only if $\forall i \in\{0, \ldots, n-1\}, \exists x \in X_{i+1}$ s.t. $\left(X_{i}, x, l\right) \in \mathcal{P}$ and $w\left(\left(X_{i}, x, l\right)\right)>0$, where $l \in\{$ att, supp $\}$.

We can now define the notion of cycle.

Definition 4 (Cycle). A path $\left\langle X_{0}, X_{1}, \ldots, X_{n}\right\rangle$ is called a cycle in $\mathcal{A S}=$ $\langle\mathcal{A}, \mathcal{P}, w\rangle$ iff $\exists x \in X_{0}$ s.t. $\left(X_{n}, x, l\right) \in \mathcal{P}$, where $l \in\{$ att, supp $\}$. 
A semantics is a function assigning an overall strength from $[0,1]$ to each argument of the WBSETAF.

Definition 5 (Semantics). Let $\mathcal{A S}=\langle\mathcal{A}, \mathcal{P}, w\rangle$ be a weighed bipolar SETAF. $A$ semantics is a function $\sigma$ transforming any $\mathcal{A S} \in \mathcal{X}_{\text {setaf }}$ into a function $\sigma_{\mathcal{A S}}: \mathcal{A} \rightarrow[0,1]$.

When $\mathcal{A S}$ is clear from the context, we simply write $\sigma(a)$ instead of $\sigma_{\mathcal{A S}}(a)$.

Definition 6 (Isomorphism). Let $\mathcal{A S}=\langle\mathcal{A}, \mathcal{P}, w\rangle$ and $\mathcal{A S}^{\prime}=\left\langle\mathcal{A}^{\prime}, \mathcal{P}^{\prime}, w^{\prime}\right\rangle$ be two WBSETAFs. We say that $f$ is an isomorphism from $\mathcal{A}$ to $\mathcal{A}^{\prime}$ w.r.t. $\mathcal{A S}$ and $\mathcal{A S}^{\prime}$ iff all the following conditions are satisfied: (1) $\forall X \subseteq \mathcal{A}, a \in \mathcal{A}$, $z \in\{$ att, supp $\},(X, a, z) \in \mathcal{P}$ iff $(f(X), f(a), z) \in \mathcal{P}^{\prime}$, (2) $\forall X \subseteq \mathcal{A}, a \in \mathcal{A}$ and $z \in\{a t t$, supp $\}$ s.t. $(X, a, z) \in \mathcal{P}, w((X, a, z))=w^{\prime}((f(X), f(a), z))$ and (3) $\forall a \in \mathcal{A}, w(a)=w^{\prime}(f(a))$

\section{Desirable Properties}

We now extend the desirable properties that a semantics should satisfy [1] for the more general case of WBSETAFs . In each set of attacking (resp. supporting) arguments, all the components are necessary. Thus, we follow an important intuition from the literature [22] (reflected in our new properties) which is that the strength of a set of arguments should be equal to the strength of the weakest argument. Of course, this is not the only intuition and other aggregation functions can be used without loss of generality.

The first property states that the overall strength returned by the semantics should only be computed based on the structural elements.

Property 1 (Anonymity). A semantics $\sigma$ satisfies anonymity iff for any two WBSETAFs $\mathcal{A S}=\langle\mathcal{A}, \mathcal{P}, w\rangle$ and $\mathcal{A S}^{\prime}=\left\langle\mathcal{A}^{\prime}, \mathcal{P}^{\prime}, w^{\prime}\right\rangle$, for any isomorphism $f$ from $\mathcal{A}$ to $\mathcal{A}^{\prime}$ w.r.t. $\mathcal{A S}$ and $\mathcal{A S}^{\prime}$, it holds that $\forall a \in \mathcal{A}, \sigma_{\mathcal{A S}}(a)=\sigma_{A S^{\prime}}(f(a))$.

The independence property states that overall strength of an argument should not be affected by any other arguments that are not connected to it.

Property 2 (Independence). A semantics $\sigma$ satisfies independence iff for any two WBSETAFs $\mathcal{A S}=\langle\mathcal{A}, \mathcal{P}, w\rangle$ and $\mathcal{A S}^{\prime}=\left\langle\mathcal{A}^{\prime}, \mathcal{P}^{\prime}, w^{\prime}\right\rangle$ s.t. $\mathcal{A} \cap \mathcal{A}^{\prime}=\emptyset$, it holds that $\forall a \in \mathcal{A}, \sigma_{\mathcal{A S}}(a)=\sigma_{\mathcal{A S} \oplus \mathcal{A} \mathcal{S}^{\prime}}(a)$.

The directionality property states that the overall strength of an argument should depend only on the significant paths directed to it.

Property 3 (Directionality). A semantics $\sigma$ satisfies directionality iff for any two WBSETAFs $\mathcal{A S}=\langle\mathcal{A}, \mathcal{P}, w\rangle$ and $\mathcal{A S}^{\prime}=\left\langle\mathcal{A}, \mathcal{P}^{\prime}, w^{\prime}\right\rangle$ s.t. all the following conditions are satisfied:

$$
\text { - } \forall y \in \mathcal{A} \cup \mathcal{P}, w(y)=w^{\prime}(y),
$$


- $\forall x, b \in \mathcal{A}, \forall X \subseteq \mathcal{A}$, if $\mathcal{P}^{\prime}=\mathcal{P} \cup\{(X, b, z)\}$, where $z \in\{a t t$, supp $\}$ and there is no $X^{\prime} \subseteq \mathcal{A}$ s.t. $b \in X^{\prime}$ and there is a significant path from $X^{\prime}$ to $x$,

then $\sigma_{\mathcal{A S}}(x)=\sigma_{\mathcal{A} \mathcal{S}^{\prime}}(x)$.

The equivalence property states that the overall strength of an argument depends only on the overall strength of its direct attackers and supporters and the weight of the corresponding relations.

Property 4 (Equivalence). A semantics $\sigma$ satisfies equivalence iff for every WBSETAF $\mathcal{A S}=\langle\mathcal{A}, \mathcal{P}, w\rangle$ and $\forall a, b \in \mathcal{A}$, if all the following conditions are satisfied:

$-w(a)=w(b)$,

- there exists a bijection $f$ between $\operatorname{Att}(a)$ and $\operatorname{Att}(b)$ s.t. $\forall X \in \operatorname{Att}(a)$, $\min _{x \in X} \sigma_{\mathcal{A S}}(x)=\min _{x^{\prime} \in f(X)} \sigma_{\mathcal{A S}}\left(x^{\prime}\right)$ and $w((X, a, a t t))=w((f(X), b, a t t))$,

- there exists a bijection $f$ between $\operatorname{Supp}(a)$ and $\operatorname{Supp}(b)$ s.t. $\forall X \in \operatorname{Supp}(a)$, $\min _{x \in X} \sigma_{\mathcal{A S}}(x)=\min _{x^{\prime} \in f(X)} \sigma_{\mathcal{A S}}\left(x^{\prime}\right)$ and $w((X, a$, supp $))=w((f(X), b$, supp $))$,

then $\sigma_{\mathcal{A S}}(a)=\sigma_{\mathcal{A S}}(b)$.

The reinforcement property states that an argument becomes stronger if the quality of its attackers is reduced or the quality of its supporters is increased.

Property 5 (Reinforcement). A semantics $\sigma$ satisfies reinforcement iff for every WBSETAF $\mathcal{A S}=\langle\mathcal{A}, \mathcal{P}, w\rangle, \forall C, C^{\prime} \subseteq 2^{\mathcal{A}}, \forall a, b \in \mathcal{A}$ and $\forall X_{1}, X_{2}, Y_{1}, Y_{2} \subseteq \mathcal{A}$ s.t. $X_{i}, Y_{i} \notin C \cup C^{\prime}$, if all the following conditions are satisfied:

- $w(a)=w(b), \operatorname{Att}(a)=C \cup\left\{X_{1}\right\}$ and $\operatorname{Att}(b)=C \cup\left\{Y_{1}\right\}$,

- $\operatorname{Supp}(a)=C^{\prime} \cup\left\{X_{2}\right\}$ and $\operatorname{Supp}(b)=C^{\prime} \cup\left\{Y_{2}\right\}$,

$-\min _{x_{1} \in X_{1}} \sigma_{\mathcal{A S}}\left(x_{1}\right) \leq \min _{y_{1} \in Y_{1}} \sigma_{\mathcal{A S}}\left(y_{1}\right)$

- $w\left(\left(X_{1}, a, a t t\right)\right) \leq w\left(\left(Y_{1}, a, a t t\right)\right)$,

$-\min _{x_{2} \in X_{2}} \sigma_{\mathcal{A S}}\left(x_{2}\right) \geq \min _{y_{2} \in Y_{2}} \sigma_{\mathcal{A S}}\left(y_{2}\right)$,

- $w\left(\left(X_{2}, a, \operatorname{supp}\right)\right) \geq w\left(\left(Y_{2}, a, \operatorname{supp}\right)\right)$,

then $\sigma_{\mathcal{A S}}(a) \geq \sigma_{\mathcal{A S}}(b)$.

The stability property states that if an argument is neither attacked nor supported, then its overall strength should be equal to its intrinsic weight.

Property 6 (Stability). A semantics $\sigma$ satisfies stability iff, for every WBSETAF $\mathcal{A S}=\langle\mathcal{A}, \mathcal{P}, w\rangle$, for every argument $a \in \mathcal{A}$, if $\operatorname{Att}(a)=\operatorname{Supp}(a)=\emptyset$, then $\sigma_{\mathcal{A S}}(a)=w(a)$.

In the original work of [1], it is assumed that the neutral overall strength is the minimum possible value, which is zero. The intuition is that the "worthless" arguments, i.e. those with the strength zero, do not contain any valuable information and thus should not impact the other arguments. In their initial work, [13] generalised the existing approaches by allowing for the neutral overall 
strength to be any value. In the later paper [14], the authors changed the neutral overall strength to be zero but left the overall strength value to be any interval. In our paper, we choose the overall strength of all semantics to be in $[0,1]$ to align with the literature standards. As a result, the neutral overall strength is chosen in the aforementioned interval.

The neutrality property states that the overall strength of an argument should not be affected by sets of attacking/supporting arguments containing arguments with an overall strength equal to the neutral overall strength.

Property 7 (Neutrality). A semantics $\sigma$ satisfies neutrality iff, there exists a unique $\alpha \in[0,1]$ s.t. both of the following conditions are satisfied:

- there exists a WBSETAF $\mathcal{A S}=\langle\mathcal{A}, \mathcal{P}, w\rangle$ and $a \in \mathcal{A}$ s.t. $\sigma_{\mathcal{A S}}(a)=\alpha$,

- for every WBSETAF $\mathcal{A S}=\langle\mathcal{A}, \mathcal{P}, w\rangle$, for every argument $a, b \in \mathcal{A}$ s.t. $w(a)=w(b), X \subseteq \mathcal{A}$ s.t. $\operatorname{Att}(a) \subseteq \operatorname{Att}(b), \operatorname{Supp}(a) \subseteq \operatorname{Supp}(b)$ and $\operatorname{Att}(a) \cup$ $\operatorname{Supp}(a) \cup\{X\}=\operatorname{Att}(b) \cup \operatorname{Supp}(b)$, if $\min _{x \in X} \sigma_{\mathcal{A S}}(x)=\alpha$ or $w((X, b, z))=0$, where $z \in\{$ att, supp $\}$,

then $\sigma_{\mathcal{A S}}(a)=\sigma_{\mathcal{A S}}(b)$.

The first item of the neutrality property is necessary because if the semantics $\sigma$ never assigns an overall strength $\beta \in[0,1]$ to an argument then neutrality is trivially satisfied by putting $\alpha=\beta$ (under the condition that whenever the intrinsic weight of an attack/support is equal to zero, it does not affect the overall strength of its target). If $\sigma$ satisfies the neutrality property, $\alpha$ is called the neutral overall strength.

The monotonicity property states that if two arguments $a$ and $b$ have the same intrinsic weight and $a$ is "less attacked" and "more supported" than $b$, then $a$ should have a higher overall strength than $b$.

Property 8 (Monotonicity). A semantics $\sigma$ satisfies monotonicity iff, for every WBSETAF $\mathcal{A S}=\langle\mathcal{A}, \mathcal{P}, w\rangle, \forall a, b \in \mathcal{A}$ s.t. $w(a)=w(b), \operatorname{Att}(a) \subseteq \operatorname{Att}(b)$ and $\forall X \in \operatorname{Att}(a), w((X, a, a t t)) \leq w((X, b, a t t)), \operatorname{Supp}(b) \subseteq \operatorname{Supp}(a)$ and $\forall X \in$ $\operatorname{Supp}(b), w((X, b, \operatorname{supp})) \leq w((X, a, \operatorname{supp}))$, then $\sigma_{\mathcal{A S}}(a) \geq \sigma_{\mathcal{A S}}(b)$.

The resilience property states that if the intrinsic weight of an argument is not 0 nor 1 then its overall strength cannot be 0 nor 1 .

Property 9 (Resilience). A semantics $\sigma$ satisfies resilience iff, for every WB$\mathrm{SETAF} \mathcal{A S}=\langle\mathcal{A}, \mathcal{P}, w\rangle, \forall a \in \mathcal{A}$, if $0<w(a)<1$ then $0<\sigma_{\mathcal{A S}}(a)<1$.

Franklin states that an attacker and a supporter of equal strength should counter-balance each other. Thus, the overall strength should remain unchanged.

Property 10 (Franklin). A semantics $\sigma$ satisfies Franklin iff, for every WBSETAF $\mathcal{A S}=\langle\mathcal{A}, \mathcal{P}, w\rangle, \forall a, b \in \mathcal{A}$ and $X, Y \subseteq \mathcal{A}$, if all of the following conditions are satisfied:

$$
-w(a)=w(b), w((X, a, a t t))=w((Y, a, \text { supp }))
$$




$$
\begin{aligned}
& -\operatorname{Att}(a)=\operatorname{Att}(b) \cup\{X\}, \operatorname{Supp}(a)=\operatorname{Supp}(b) \cup\{Y\}, \\
& -\min _{x \in X} \sigma_{\mathcal{A S}}(x)=\min _{y \in Y} \sigma_{\mathcal{A S}}(y),
\end{aligned}
$$

then $\sigma_{\mathcal{A S}}(a)=\sigma_{\mathcal{A S}}(b)$.

The strengthening property states that if an argument receives more supports than attacks, then its overall strength should increase. Roughly speaking, there are three cases: (1) there are more supporters than attackers, (2) the weakest strength of a set attack is strictly weaker than the weakest strength of the corresponding supporter, (3) the intrinsic weight of one attack is strictly weaker than the corresponding support.

Property 11 (Strengthening). A semantics $\sigma$ satisfies strengthening iff, for every WBSETAF $\mathcal{A S}=\langle\mathcal{A}, \mathcal{P}, w\rangle, \forall a \in \mathcal{A}$, if $w(a)<1$ and there exists an injective function $f$ from $\operatorname{Att}(a)$ to $\operatorname{Supp}(a)$ s.t. $\forall X \in \operatorname{Att}(a), \min _{x \in X} \sigma_{\mathcal{A S}}(x) \leq$ $\min _{x^{\prime} \in f(X)} \sigma_{\mathcal{A S}}\left(x^{\prime}\right), \forall X \in \operatorname{Att}(a), w((X, a, a t t)) \leq w((f(X), a$, supp $))$ and at least one of the following conditions is satisfied:

- there exists $X^{\prime} \in \operatorname{Supp}(a) \backslash\{f(X) \mid X \in \operatorname{Att}(a)\}$ such that $w\left(\left(X^{\prime}, a\right.\right.$, supp $\left.)\right)>$ 0 and $\min _{x^{\prime} \in X^{\prime}} \sigma_{\mathcal{A S}}\left(x^{\prime}\right)>0$

- there exists $X \in \operatorname{Att}(a)$ such that $\min _{x \in X} \sigma_{\mathcal{A S}}(x)<\min _{x^{\prime} \in f(X)} \sigma_{\mathcal{A S}}\left(x^{\prime}\right)$ and $w((f(X), a$, supp $))>0$, or

- there exists $X \in \operatorname{Att}(a)$ such that $w((X, a, a t t))<w((f(X), a$, supp $))$ and $\min _{x^{\prime} \in f(X)} \sigma_{\mathcal{A S}}\left(x^{\prime}\right)>0$

then $\sigma_{\mathcal{A S}}(a)>w(a)$.

Similarly, the weakening property states that if an argument receives less supports than attacks, then its overall strength should decrease.

Property 12 (Weakening). A semantics $\sigma$ satisfies weakening iff, for every WBSETAF $\mathcal{A S}=\langle\mathcal{A}, \mathcal{P}, w\rangle, \forall a \in \mathcal{A}$, if $0<w(a)$ and there exists an injective function $f$ from $\operatorname{Supp}(a)$ to $\operatorname{Att}(a)$ s.t. $\forall X \in \operatorname{Supp}(a), \min _{x \in X} \sigma_{\mathcal{A S}}(x) \leq \min _{x^{\prime} \in f(X)} \sigma_{\mathcal{A S}}\left(x^{\prime}\right)$, $\forall X \in \operatorname{Supp}(a), w((X, a, \operatorname{supp})) \leq w((f(X), a, a t t))$ and at least one of the following conditions is satisfied:

- there exists $X^{\prime} \in \operatorname{Att}(a) \backslash\{f(X) \mid X \in \operatorname{Supp}(a)\}$ such that $w\left(\left(X^{\prime}, a, a t t\right)\right)>0$ and $\min _{x^{\prime} \in X^{\prime}} \sigma_{\mathcal{A S}}\left(x^{\prime}\right)>0$,

- there exists $X \in \operatorname{Supp}(a)$ such that $\min _{x \in X} \sigma_{\mathcal{A S}}(x)<\min _{x^{\prime} \in f(X)} \sigma_{\mathcal{A S}}\left(x^{\prime}\right)$ and $w((f(X), a, a t t))>0$, or

- there exists $X \in \operatorname{Supp}(a)$ such that $w((X, a$, supp $))<w((f(X), a, a t t))$ and $\min _{x^{\prime} \in f(X)} \sigma_{\mathcal{A S}}\left(x^{\prime}\right)>0$

then $\sigma_{\mathcal{A S}}(a)<w(a)$. 
The next property states that adding an attack to an argument should decrease its overall strength whereas adding a support should increase it. The idea of accumulation is similar to that of monotonicity except that monotonicity is defined on two arguments of the same graph whereas accumulation is defined on the "same" argument in the "copy" of the graph. Note that in the next section, we identify the conditions under which monotonicity and accumulation are equivalent (Corollary 1).

Property 13 (Accumulation). A semantics $\sigma$ satisfies accumulation iff, for every two WBSETAFs $\mathcal{A S}=\langle\mathcal{A}, \mathcal{P}, w\rangle$ and $\mathcal{A} \mathcal{S}^{\prime}=\left\langle\mathcal{A}, \mathcal{P}^{\prime}, w^{\prime}\right\rangle, \forall a \in \mathcal{A}, \forall X \in 2^{\mathcal{A}}$, if $\forall p \in \mathcal{A} \cup \mathcal{P}$, we have $w(p)=w^{\prime}(p)$, and $\mathcal{P}^{\prime}=\mathcal{P} \cup\{(X, a, z)\}$ then $(1) \sigma_{\mathcal{A S}^{\prime}}(a) \leq$ $\sigma_{\mathcal{A S}}(a)$ if $z=a t t$ and $(2) \sigma_{\mathcal{A S}}(a) \geq \sigma_{\mathcal{A S}}(a)$ if $z=$ supp.

\section{Links Between Properties}

We now study the link between the properties that we previously defined.

Proposition 1. Let $\sigma$ be a semantics that satisfies independence, directionality, stability, accumulation. Then, $\sigma$ satisfies monotonicity.

Proof. Let $\mathcal{A S}=\langle\mathcal{A}, \mathcal{P}, w\rangle$ and $a, b \in \mathcal{A}$ s.t. the conditions of monotonicity are satisfied. The intuition of the next construction is the following. We will define a "copy" $\mathcal{A S}$ ' of $\mathcal{A S}$. For each argument $x$, we create its copy $x^{\prime}$. Furthermore, we keep only the attacks from the direct attackers of $a$ and $b$ and only the supports from the direct supporters of $a$ and $b$. All the other attacks and supports are forgotten. This is done to allow for the application of directionality when comparing $\mathcal{A} \mathcal{S}^{\prime}$ and $\mathcal{A} \mathcal{S}^{1}$ (which will be defined later in the proof).

Let us define $\mathcal{A} \mathcal{S}^{\prime}=\left\langle\mathcal{A}^{\prime}, \mathcal{P}^{\prime}, w^{\prime}\right\rangle$ s.t. there exists a bijection $f: \operatorname{Att}(b) \cup$ $\operatorname{Supp}(a) \cup\{a, b\} \rightarrow \mathcal{A}^{\prime}$ defined as $\forall x, f(x)=x^{\prime}$ and:

- $\mathcal{A}^{\prime}=\left\{a^{\prime}, b^{\prime}\right\} \cup\left\{x_{i}^{\prime} \mid x_{i} \in X\right.$ and $\left.X \in \operatorname{Att}(b)\right\} \cup\left\{y_{i}^{\prime} \mid y_{i} \in Y\right.$ and $\left.Y \in \operatorname{Supp}(a)\right\}$.

$-\mathcal{P}^{\prime}=\{(f(X), f(a), a t t)$ such that $(X, a, a t t) \in \mathcal{P}\} \cup\{(f(X), f(b), a t t)$ such that $(X, b, a t t) \in \mathcal{P}\} \cup\{(f(Y), f(a)$, supp $)$ such that $(Y, a$, supp $) \in \mathcal{P}\} \cup$ $\{(f(Y), f(b)$, supp $)$ such that $(Y, b$, supp $) \in \mathcal{P}\}$.

- Let us define $w^{\prime}$ as follows: $w^{\prime}(f(a))=w(a), w^{\prime}(f(b))=w(b), \forall x \in \mathcal{A}^{\prime} \backslash$ $\left\{a^{\prime}, b^{\prime}\right\}, w^{\prime}(x)=\sigma_{\mathcal{A S}}(x)$ and $\forall(T, t, z) \in \operatorname{Att}(b) \cup \operatorname{Supp}(a)$, let $w^{\prime}((f(T), f(t), z))=w((T, t, z))$.

From stability, we know that $\forall t^{\prime} \in \mathcal{A}^{\prime} \backslash\left\{a^{\prime}, b^{\prime}\right\}$, we have $\sigma_{\mathcal{A S}}\left(t^{\prime}\right)=w^{\prime}\left(t^{\prime}\right)=$ $\sigma_{\mathcal{A S}}(t)$. By using the independence and equivalence properties, we have that $\sigma_{\mathcal{A S}}(a)=\sigma_{\mathcal{A S}^{\prime}}\left(a^{\prime}\right)$ and $\sigma_{\mathcal{A S}}(b)=\sigma_{\mathcal{A S}^{\prime}}\left(b^{\prime}\right)$. Let us use the notation $\operatorname{Att}\left(a^{\prime}\right)=$ $\left\{X_{1}^{\prime}, \ldots, X_{n}^{\prime}\right\}, \operatorname{Att}\left(b^{\prime}\right)=\operatorname{Att}\left(a^{\prime}\right) \cup\left\{X_{n+1}^{\prime}, \ldots, X_{m}^{\prime}\right\}, \operatorname{Supp}\left(b^{\prime}\right)=\left\{Y_{1}^{\prime}, \ldots, Y_{k}^{\prime}\right\}$ and $\operatorname{Supp}\left(a^{\prime}\right)=\operatorname{Supp}\left(b^{\prime}\right) \cup\left\{Y_{k+1}^{\prime}, \ldots, Y_{l}^{\prime}\right\}$.

Let us now remove attacks from $A t t\left(b^{\prime}\right) \backslash A t t\left(a^{\prime}\right)$ to $b^{\prime}$ and supports from $\operatorname{Supp}\left(a^{\prime}\right) \backslash \operatorname{Supp}\left(b^{\prime}\right)$ to $a^{\prime}$ using the following procedures. 
- Let $\mathcal{A S}^{1}=\left\langle\mathcal{A}^{\prime}, \mathcal{P}^{1}, w^{1}\right\rangle$ with $\mathcal{P}^{1}=\mathcal{P}^{\prime} \backslash\left\{\left(X_{m}^{\prime}, b^{\prime}, a t t\right)\right\}$ and $\forall p \in \mathcal{A}^{\prime} \cup$ $\mathcal{P}^{1}, w^{1}(p)=w^{\prime}(p)$. By accumulation, we have that $\sigma_{\mathcal{A} \mathcal{S}^{1}}\left(b^{\prime}\right) \geq \sigma_{\mathcal{A} \mathcal{S}^{\prime}}\left(b^{\prime}\right)$. Moreover, by directionality, $\forall t \in \mathcal{A}^{\prime}, \sigma_{\mathcal{A S}^{1}}(t)=\sigma_{\mathcal{A} \mathcal{S}^{\prime}}(t)$. We repeat this procedure $m-n$ times to remove all the attacks from $\operatorname{Att}\left(b^{\prime}\right) \backslash \operatorname{Att}\left(a^{\prime}\right)$ to $b^{\prime}$. Let us call the resulting graph $\mathcal{A S}^{m-n}$.

- Let us define $\mathcal{A S}^{m-n+1}=\left\langle\mathcal{A}^{\prime}, \mathcal{P}^{m-n+1}, w^{m-n+1}\right\rangle$ with $\mathcal{P}^{m-n+1}=\mathcal{P}^{m-n} \backslash$ $\left\{\left(Y_{l}^{\prime}, a^{\prime}\right.\right.$, supp $\left.)\right\}$ and $\forall p \in \mathcal{A}^{\prime} \cup \mathcal{P}^{m-n+1}, w^{m-n+1}(p)=w^{\prime}(p)$. By accumulation, we have that $\sigma_{\mathcal{A S}}{ }^{m-n+1}\left(a^{\prime}\right) \leq \sigma_{\mathcal{A S}^{m-n}}\left(a^{\prime}\right)$. Moreover, by directionality, $\forall t \in \mathcal{A}^{\prime}, \sigma_{\mathcal{A S}^{m-n}}(t)=\sigma_{\mathcal{A} \mathcal{S}^{m-n+1}}(t)$. We repeat this procedure $l-k$ times to remove all the supports from $\operatorname{Supp}\left(a^{\prime}\right) \backslash \operatorname{Supp}\left(b^{\prime}\right)$ to $a^{\prime}$. Let us call the resulting graph $\mathcal{A S}^{*}$.

By equivalence, we have that $\sigma_{\mathcal{A} \mathcal{S}^{*}}(a)=\sigma_{\mathcal{A S}^{*}}(b)$. Thus:

$$
\begin{aligned}
\sigma_{\mathcal{A S}^{\prime}}\left(b^{\prime}\right) \leq \sigma_{\mathcal{A S}^{1}}\left(b^{\prime}\right) \leq \cdots \leq \sigma_{\mathcal{A S}^{*}}\left(b^{\prime}\right) & = \\
& \sigma_{\mathcal{A S}} \mathcal{S}^{*}\left(a^{\prime}\right) \leq \cdots \leq \sigma_{\mathcal{A S}}\left(a^{\prime}\right) \leq \sigma_{\mathcal{A S}}\left(a^{\prime}\right)
\end{aligned}
$$

Finally, since we have that $\sigma_{\mathcal{A S}}(b)=\sigma_{\mathcal{A} \mathcal{S}^{\prime}}\left(b^{\prime}\right)$ and $\sigma_{\mathcal{A S}}(a)=\sigma_{\mathcal{A S}}\left(a^{\prime}\right)$, we conclude that $\sigma_{\mathcal{A S}}(a) \geq \sigma_{\mathcal{A S}}(b)$.

Regarding the previous result, the proposition holds for an arbitrary semantics; note also that if $\sigma$ is a semantics that is defined only on acyclic graphs, we can show that $\sigma$ satisfies monotonocity (on the class of acyclic graphs).

Proposition 2. Let $\sigma$ be a semantics that satisfies anonymity, independence, directionality, equivalence and monotonicity on the class of acyclic graphs. Then, $\sigma$ satisfies accumulation on the class of acyclic graphs.

Proposition 1 also holds for a semantics $\sigma$ defined on acyclic graphs only. Hence, from that fact and Proposition 2, we conclude that accumulation and monotonicity are equivalent given anonymity, independence, directionality, stability and equivalence. We formally state this in the next corollary.

Corollary 1. Let $\sigma$ be a semantics that satisfies anonymity, independence, directionality, stability and equivalence on the class of acyclic graphs. Then $\sigma$ satisfies accumulation on the class of acyclic graphs iff it satisfies monotonicity on the class of acyclic graphs.

\section{Gradual Semantics}

As mentioned before, in each set of attacking (resp. supporting) arguments, all the components are necessary. Thus, removing one argument from the set would make the attack (resp. support) void. In this section, we generalise the semantics from the literature by considering the force of the set of attacking (resp. supporting) arguments to be the force of the weakest argument of the set. For simplicity, we also incorporate the intrinsic weight of attacks by means of the multiplication operator but the whole approach can be generalised with other aggregating 
methods. Moreover, the first two semantics (Euler-based and DF-Quad) do not always converge in the class of cyclic WBSETAFs. For this reason, as usual in the literature $[1,20]$, we consider that the class of all acyclic WBSETAFs is still expressive enough to deserve attention. In the next definitions, we generalise the existing Euler-based [1] and DF-Quad semantics [20] for the new framework.

Definition 7 (Euler-based semantics). Let $\mathcal{A S}=\langle\mathcal{A}, \mathcal{P}, w\rangle \in \mathcal{X}_{\text {setaf }}$ be an acyclic WBSETAF. $\forall a \in \mathcal{A}$, we have:

$$
\sigma_{\mathcal{A S}}^{E B}(a)=1-\frac{1-w(a)^{2}}{1+w(a) e^{E(a)}}
$$

where $E(a)=Y-Z$ with $Y=\sum_{X \in \operatorname{Supp}(a)} \min _{x \in X} \sigma_{\mathcal{A} \mathcal{S}}^{E B}(x) \cdot w((X, a$, supp $))$ and $Z=$ $\sum_{X \in A t t(a)} \min _{x \in X} \sigma_{\mathcal{A} \mathcal{S}}^{E B}(x) \cdot w((X, a, a t t))$.

\begin{tabular}{|l|c|c|c|c|c|c|c|c|c|c|}
\hline & \multicolumn{3}{|c|}{$\mathcal{X}_{\text {setaf }}$} & \multicolumn{3}{|c|}{$\mathcal{X}_{\text {setaf }}^{>0}$} & \multicolumn{3}{|c|}{$\mathcal{X}_{\text {setaf }}^{<1}$} \\
\hline & $\sigma^{E B}$ & $\sigma^{D F}$ & $\sigma^{S D}$ & $\sigma^{E B}$ & $\sigma^{D F}$ & $\sigma^{S D}$ & $\sigma^{E B}$ & $\sigma^{D F}$ & $\sigma^{S D}$ \\
\hline Anonymity & $\checkmark$ & $\checkmark$ & $\checkmark$ & $\checkmark$ & $\checkmark$ & $\checkmark$ & $\checkmark$ & $\checkmark$ & $\checkmark$ \\
Independence & $\checkmark$ & $\checkmark$ & $\checkmark$ & $\checkmark$ & $\checkmark$ & $\checkmark$ & $\checkmark$ & $\checkmark$ & $\checkmark$ \\
Directionality & $\checkmark$ & $\checkmark$ & $\checkmark$ & $\checkmark$ & $\checkmark$ & $\checkmark$ & $\checkmark$ & $\checkmark$ & $\checkmark$ \\
Equivalence & $\checkmark$ & $\checkmark$ & $\checkmark$ & $\checkmark$ & $\checkmark$ & $\checkmark$ & $\checkmark$ & $\checkmark$ & $\checkmark$ \\
Reinforcement & $\checkmark$ & $\checkmark$ & $\checkmark$ & $\checkmark$ & $\checkmark$ & $\checkmark$ & $\checkmark$ & $\checkmark$ & $\checkmark$ \\
Stability & $\checkmark$ & $\checkmark$ & $\checkmark$ & $\checkmark$ & $\checkmark$ & $\checkmark$ & $\checkmark$ & $\checkmark$ & $\checkmark$ \\
Neutrality & $\checkmark$ & $\checkmark$ & $x$ & $x$ & $\checkmark$ & $x$ & $\checkmark$ & $\checkmark$ & $x$ \\
Monotonicity & $\checkmark$ & $\checkmark$ & $x$ & $\checkmark$ & $\checkmark$ & $x$ & $\checkmark$ & $\checkmark$ & $x$ \\
Resilience & $\checkmark$ & $x$ & $\checkmark$ & $\checkmark$ & $x$ & $\checkmark$ & $\checkmark$ & $\checkmark$ & $\checkmark$ \\
Franklin & $\checkmark$ & $x$ & $x$ & $\checkmark$ & $x$ & $x$ & $\checkmark$ & $x$ & $x$ \\
Strengthening & $x$ & $x$ & $x$ & $\checkmark$ & $x$ & $x$ & $x$ & $\checkmark$ & $x$ \\
Weakening & $x$ & $x$ & $x$ & $x$ & $x$ & $x$ & $\checkmark$ & $\checkmark$ & $x$ \\
Accumulation & $\checkmark$ & $\checkmark$ & $x$ & $\checkmark$ & $\checkmark$ & $x$ & $\checkmark$ & $\checkmark$ & $x$ \\
\hline
\end{tabular}

Table 1. Satisfaction of the properties by the gradual semantics on acyclic graphs

Definition 8 (DF-Quad semantics). Let $\mathcal{A S}=\langle\mathcal{A}, \mathcal{P}, w\rangle \in \mathcal{X}_{\text {setaf }}$ be an acyclic WBSETAF. $\forall a \in \mathcal{A}$, we have: if $v_{s}(a)=v_{a}(a), \sigma_{\mathcal{A S}}^{D F}(a)=w(a)$; else $\sigma_{\mathcal{A S}}^{D F}(a)=w(a)+\left(0.5+\frac{v_{s}(a)-v_{a}(a)}{2 \cdot\left|v_{s}(a)-v_{a}(a)\right|}-w(a)\right) \cdot\left|v_{s}(a)-v_{a}(a)\right|$,

where $v_{a}(a)=1-\prod_{X \in \operatorname{Att}(a)}\left(1-\min _{x \in X} \sigma_{\mathcal{A S}}^{D F}(x) \cdot w((X, a, a t t))\right)$ and $v_{s}(a)=$ $1-\prod_{X \in \operatorname{Supp}(a)}\left(1-\min _{x \in X} \sigma_{\mathcal{A \mathcal { S }}}^{D F}(x) \cdot w((X, a\right.$, supp $\left.))\right)$. Please note that if $\operatorname{Att}(a)=\emptyset$ then $v_{a}=0$. Similarly, $v_{s}=0$ if $\operatorname{Supp}(a)=\emptyset$. 
We also generalise the Sigmoid damped max-based gradual semantics [14] for this framework. For presentation purposes, we changed the original definition to match the other semantics by translating the range of the semantics from $[-1,1]$ to $[0,1]$. We also changed the "top" operator from the original semantics to "max" because we wanted to take all the arguments into account for determining the overall strength. Note that the sets of attacking (resp. supporting) arguments with a minimum element having a score strictly inferior to 0.5 will increase (resp. decrease) the score of the attacked (resp. supported) argument. This effect is an intrinsic aspect of the original Sigmoid damped max-based semantics, which was not introduced by the generalisation we propose.

Definition 9 (Sigmoid damped max-based semantics). Given the WB$\operatorname{SETAF} \mathcal{A S}=\langle\mathcal{A}, \mathcal{P}, w\rangle \in \mathcal{X}_{\text {setaf }}$ and $\delta>2 . \forall a \in \mathcal{A}$, we have $\sigma_{\mathcal{A} \mathcal{S}}^{S D}(a)=$ $f\left(\frac{Y(a)}{\delta}+f^{-1}(w(a))\right)$, where

$$
\begin{gathered}
Y(a)=\max _{X \in S u p p(a)}\left(\min _{x \in X} f^{-1}\left(\sigma_{\mathcal{A S}}^{S D}(x)\right) \cdot w((X, a, \text { supp }))\right) \\
-\max _{X \in A t t(a)}\left(\min _{x \in X} f^{-1}\left(\sigma_{\mathcal{A S}}^{S D}(x)\right) \cdot w((X, a, a t t))\right) \text { and } f(x)=\frac{\tanh (x)+1}{2} .
\end{gathered}
$$

In Table 1, we show the satisfaction of the properties by the aforementioned gradual semantics on the three classes $\mathcal{X}_{\text {setaf }}, \mathcal{X}_{\text {setaf }}^{<1}$ and $\mathcal{X}_{\text {setaf }}^{>0}$. Due to space limitations, we do not show all the proofs in the paper although all the combinations were diligently proved. We now provide a brief overview of the property satisfaction on the general class $\mathcal{X}_{\text {setaf }}$.

The Euler-based semantics satisfies the greatest number of properties. The only two non-satisfied properties are strengthening and weakening. Note that the only case when an argument cannot be weakened (resp. strengthened) is the case of an argument that has the maximal (resp. minimal) intrinsic weight. It seems to us that in several reasonable application contexts, this behaviour of the Euler-based semantics can be seen as rational.

The DF-Quad semantics violates strengthening and weakening as well as Franklin and resilience. Again, this is not necessarily a fatal problem, since in some contexts it might not be a good idea to be able to cancel one positive argument with one negative argument of the same strength.

Finally, the Sigmoid damped max-based semantics violates even more properties, which is (we believe) linked to its simple idea to take into account only the strongest attacker and the strongest supporter and to ignore the others. Note that Sigmoid damped max-based semantics does not satisfy neutrality, even if we use the generalised version of the proposition that allows for any number to be the neutral overall strength. Similar to the original version by Mossakowski and Neuhaus [13], we could restrict our version of Sigmoid damped max-based semantics to arguments with intrinsic strengths above 0.5 only, which would lead to satisfying neutrality. Notice that every property is satisfied by Sigmoid damped max-based semantics on the class $\mathcal{X}_{\text {setaf }}$ iff it is satisfied on $\mathcal{X}_{\text {setaf }}^{>0}$ and on $\mathcal{X}_{\text {setaf }}^{<1}$. The reason is that the Sigmoid damped max-based semantics is only defined in the interval $(0,1)$. 
Interestingly, we also showed that the new DF-Quad semantics does not satisfy Franklin (see Example 1) and that the Euler-based semantics does not satisfy strengthening on the class of non-maximal graphs even in the acyclic case (see Example 2). Since neither of our examples uses set attacks, we conclude that the original versions of those two semantics do not satisfy the aforementioned properties, contrary to what was suggested by Amgoud and Ben-Naim [1].

Example 1. Let $\mathcal{A S}=\langle\mathcal{A}, \mathcal{P}, w\rangle$ s.t. $\mathcal{A}=\left\{a, b, x_{1}, y_{1}, z\right\}, \mathcal{P}=\{(\{z\}, b, a t t)$, $(\{z\}, a, a t t),\left(\left\{y_{1}\right\}, a\right.$, supp $\left.),\left(\left\{x_{1}\right\}, a, a t t\right)\right\}, w(a)=w(b)=0.5, w\left(x_{1}\right)=w\left(y_{1}\right)=$ $0.7, w(z)=0.6$ and $w\left(\left(\left\{x_{1}\right\}, a, a t t\right)\right)=w((\{z\}, b, a t t))=w((\{z\}, a, a t t))=$ $w\left(\left(\left\{y_{1}\right\}, a\right.\right.$, supp $\left.)\right)=1$, represented in Fig. 1. We have that $v_{s}(a)=1-(1-0.7)=$ $0.7, v_{a}(a)=1-0.3 \cdot 0.4=0.88, v_{s}(b)=0$ and $v_{a}(b)=1-(1-0.6)=0.6$. As a result, $\sigma_{\mathcal{A S}}^{D F}(a)=1+(-0.5) \cdot 0.18=0.91$ whereas $\sigma_{\mathcal{A S}}^{D F}(b)=1+(-0.5) \cdot 0.6=0.7$

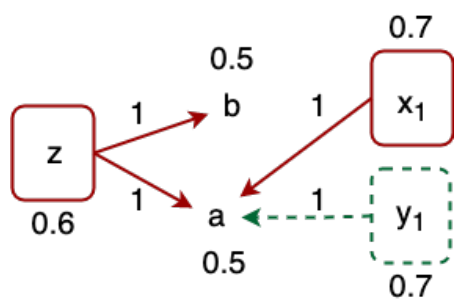

Fig. 2. Counter-example of the satisfaction of Franklin by DF-Quad.

Example 2. Let $\mathcal{A S}=\langle\mathcal{A}, \mathcal{P}, w\rangle$ s.t. $\mathcal{A}=\{a, b\}, \mathcal{P}=\{(\{b\}, a$, supp $)\}, w(a)=$ $0, w(b)=0.5$ and $w((\{b\}, a$, supp $))=1$. Then, $\sigma_{\mathcal{A} \mathcal{S}}^{E B}(a)=w(a)=0$.

\section{Discussion}

In this paper, we studied gradual semantics for WBSETAFs. This is the first study of the properties that a gradual semantics should satisfy in the framework that allows for bipolar interactions (i.e. both attacks and supports), weights (on both arguments and attacks) and SETAFs (the possibility for several arguments to jointly attack or support an argument). We generalised twelve properties from the literature $[1,14]$ and introduced a new one called "accumulation". We proved some links between the properties, and generalised three semantics from the literature to be applicable with WBSETAFs. We conducted a formal evaluation of those semantics against the properties on three classes of argumentation graphs.

We also provided an implementation of the three new semantics available at https://github.com/AnonymousConfsSubmissions/WBSETAFs. Please note that this implementation is based on an extension of the ASPARTIX format for WBSETAFs. 


\section{Bibliography}

[1] L. Amgoud and J. Ben-Naim. Weighted Bipolar Argumentation Graphs: Axioms and Semantics. In Proceedings of the Twenty-Seventh International Joint Conference on Artificial Intelligence, IJCAI 2018, 2018.

[2] L. Amgoud, J. Ben-Naim, D. Doder, and S. Vesic. Acceptability Semantics for Weighted Argumentation Frameworks. In Proceedings of the TwentySixth International Joint Conference on Artificial Intelligence, IJCAI 2017, pages 56-62, 2017.

[3] L. Amgoud, C. Cayrol, M.-C. Lagasquie-Schiex, and P. Livet. On bipolarity in argumentation frameworks. Int. J. Intell. Syst., 23(10):1062-1093, 2008.

[4] L. Amgoud and D. Doder. Gradual semantics accounting for varied-strength attacks. In Proceedings of the 18th International Conference on Autonomous Agents and MultiAgent Systems, AAMAS 2019, pages 1270-1278, 2019.

[5] T. J. M. Bench-Capon and H. Prakken. Justifying actions by accruing arguments. In Computational Models of Argument: Proceedings of COMMA 2006, pages 247-258, 2006.

[6] E. Bonzon, J. Delobelle, S. Konieczny, and N. Maudet. A Comparative Study of Ranking-Based Semantics for Abstract Argumentation. In Proceedings of the Thirtieth AAAI Conference on Artificial Intelligence, pages 914-920, 2016.

[7] C. Cayrol and M.-C. Lagasquie-Schiex. On the acceptability of arguments in bipolar argumentation frameworks. In Symbolic and Quantitative Approaches to Reasoning with Uncertainty, 8th European Conference, ECSQARU 2005, Proceedings, pages 378-389, 2005.

[8] J. Leite and J. Martins. Social Abstract Argumentation. In IJCAI 2011, Proceedings of the 22nd International Joint Conference on Artificial Intelligence, pages 2287-2292, 2011.

[9] M. J. G. Lucero, C. I. Chesñevar, and G. R. Simari. Modelling argument accrual in possibilistic defeasible logic programming. In Symbolic and Quantitative Approaches to Reasoning with Uncertainty, 10th European Conference, ECSQARU 2009, pages 131-143, 2009.

[10] M. J. G. Lucero, C. I. Chesñevar, and G. R. Simari. On the accrual of arguments in defeasible logic programming. In Proceedings of the 21st International Joint Conference on Artificial Intelligence, IJCAI 2009, pages 804-809, 2009.

[11] P.-A. Matt and F. Toni. A Game-Theoretic Measure of Argument Strength for Abstract Argumentation. In Logics in Artificial Intelligence, 11th European Conference, JELIA 2008, Proceedings, pages 285-297, 2008.

[12] S. Modgil and T. J. M. Bench-Capon. Integrating dialectical and accrual modes of argumentation. In Computational Models of Argument: Proceedings of COMMA 2010, September 8-10, 2010, pages 335-346, 2010.

[13] T. Mossakowski and F. Neuhaus. Bipolar Weighted Argumentation Graphs. CoRR, abs/1611.08572, 2016. 
[14] T. Mossakowski and F. Neuhaus. Modular Semantics and Characteristics for Bipolar Weighted Argumentation Graphs. CoRR, abs/1807.06685, 2018.

[15] S. H. Nielsen and S. Parsons. A Generalization of Dung's Abstract Framework for Argumentation: Arguing with Sets of Attacking Arguments. In N. Maudet, S. Parsons, and I. Rahwan, editors, Argumentation in MultiAgent Systems, pages 54-73. Springer Berlin Heidelberg, 2007.

[16] C. d. C. Pereira, A. Tettamanzi, and S. Villata. Changing One's Mind: Erase or Rewind? In Proceedings of the 22nd International Joint Conference on Artificial Intelligence, IJCAI 2011, pages 164-171, 2011.

[17] N. Potyka. Continuous dynamical systems for weighted bipolar argumentation. In Principles of Knowledge Representation and Reasoning: Proceedings of the Sixteenth International Conference, KR 2018, pages 148-157, 2018.

[18] H. Prakken. A study of accrual of arguments, with applications to evidential reasoning. In Proceedings of the Tenth International Conference on Artificial Intelligence and Law, ICAIL 2005, pages 85-94, 2005.

[19] H. Prakken. Modelling accrual of arguments in ASPIC+. In Proceedings of the Seventeenth International Conference on Artificial Intelligence and Law, ICAIL 2019, pages 103-112, 2019.

[20] A. Rago, F. Toni, M. Aurisicchio, and P. Baroni. Discontinuity-Free Decision Support with Quantitative Argumentation Debates. In Principles of Knowledge Representation and Reasoning: Proceedings of the Fifteenth International Conference, KR 2016, pages 63-73, 2016.

[21] B. Verheij. Accrual of arguments in defeasible argumentation. In Dutch/German Workshop on Nonmonotonic Reasoning. Proceedings of the Second Workshop, Delft University of Technology, Universiteit Utrecht, pages 217-224, 1995.

[22] B. Yun, S. Vesic, and M. Croitoru. Ranking-based semantics for sets of attacking arguments. In The Thirty-Fourth AAAI Conference on Artificial Intelligence, AAAI 2020, pages 3033-3040, 2020. 